\title{
Tyrosine-priming Modulates Phenylpropanoid Pathway in Maize Grown under Different pH Regimes
}

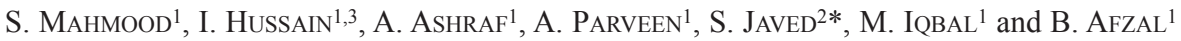 \\ ${ }^{1}$ Department of Botany, Government College University, Faisalabad, Pakistan \\ ${ }^{2}$ Department of Applied Chemistry and Biochemistry, Government College University, Faisalabad, Pakistan \\ ${ }^{3}$ Department of Environmental Life Sciences, Graduate School of Life Sciences, Tohoku University, Sendai, \\ Japan
}

(Received 12 May 2016; Accepted 29 August 2016;

Communicated by A. Pécsváradi)

\begin{abstract}
Alkaline and acidic $\mathrm{pH}$ of soil limit crop yield. Products of phenylpropanoid pathway play a key part in plant abiotic stress tolerance. It was aimed to assess efficacy of tyrosinepriming for activation of enzyme involved in phenolic accumulation induction of $\mathrm{pH}$ tolerance in maize seedlings. Seeds of two maize cultivars, namely Sadaf ( $\mathrm{pH}$ tolerant) and $\mathrm{S}-2002$ ( $\mathrm{pH}$ sensitive), were grown under three $\mathrm{pH}$ levels (3, 7 and 11). Eight and twelve days old seedlings were harvested and parted into roots and shoots for the assessment of growth, enzymatic and non-enzymatic antioxidants. PAL activity was directly correlated with total soluble phenolics, flavonoids, growth and seedling vigour. Lower accumulation of phenolics and PAL activity in the $\mathrm{pH}$ sensitive (S-2002) cultivar indicated greater oxidative damage caused by $\mathrm{pH}$ extremes. Priming improved antioxidative potential by enhancing PAL activity and phenolics accumulation and hence increased growth in maize seedlings.
\end{abstract}

Keywords: maize, phenylpropanoid, enzymes, phenolics, $\mathrm{pH}$

Abbreviations: PAL: Phenylalanine ammonia lyase, TSP: Total soluble phenolics, CAT: Catalase, FLV: Flavonoids, LA: Leaf area

\section{Introduction}

In nature, low and high $\mathrm{pH}$ levels are experienced by plants growing in environment. For survival in alkaline range of $\mathrm{pH}$ in the rooting medium, plants need maintenance of stable intracellular $\mathrm{pH}$ (Yang et al. 2007, 2008a, c) that indicates the importance of $\mathrm{pH}$ adjustments as a component of plant alkali tolerance potential. High $\mathrm{pH}(<7)$ alone or with salinity negatively affects grasslands and farming lands (Deng et al. 2006). The high $\mathrm{pH}$ in the rooting medium directly contribute in the precipitation of some ions including $\mathrm{Ca}^{2+}$ and $\mathrm{Mg}^{2+}$ that negatively affect plant functioning including injures in the root system (Yang et al. 2008b). Alkaline range of $\mathrm{pH}$ promotes osmotic stress and ion toxicity (de Lacerda et al. 2003).

Soil acidity is limiting global crop yield (Samac and Tesfaye 2003). Globally acid affected area is continually increasing where a number of factors are contributing for phytotoxic activity of the soil. Over $50 \%$ of non-irrigated, arable land on $\mathrm{pH}$ level lower

*Corresponding author; E-mail: drsadiajaved@yahoo.com 
than 7, particularly in the tropical areas where higher level of rainfall results in the prolongation of soluble base leaching (Kidd and Proctor 2001).

Unfavourable circumstances induced by unfavourable $\mathrm{pH}$ range (both high and low) along with production of reactive oxygen species (ROS) in plants impair. Acidity alters the availability of cations on the sites of root absorption. Furthermore, $\mathrm{H}^{+}$interferes with ion uptake and transportation leading to the root membrane leakage (Foy 1992).

Under steady-state conditions, the ROS molecules are scavenged by various antioxidative defense mechanisms (Foyer and Noctor 1992). Metabolic adjustment is one of these mechanisms, which is gifted with the accumulation a number of primary and secondary metabolites. It assists plants to cope with the challenges imposed by stressful environmental conditions. End products of the shikimate pathway (phenylalanine, tyrosine and tryptophan) serve as precursors of phenylpropanoid pathway (Tzin and Galili 2010). Phenylalanine ammonia-lyase (EC 4.3.1.5) catalyzes the crucial step in phenylpropanoid metabolism (Raes et al. 2003). This pathway in plants produces an immense collection of compounds including phenolics, indoleglucosinolates, flavonoids, hydroxycinnamic acids, lignins, and lignans (Tzin and Galili 2010). Phenolic compounds secure the plants from biotic and abiotic stresses combating ROS produced by the stresses (Rice-Evans 2001). Thus activation of the phenolic metabolism may enhance stress tolerance via accumulation of the products of phenylpropanoid pathway. It also contributes towards structural support and stress tolerance (Vogt 2010; Pawlak-Sprada et al. 2011). The expression of PAL is species-specific. Like other metabolic enzymes, it could be regulated allosterically by the availability of some of its substrates or the products of its relevant pathway (Goodey and Benkovic 2008; Huang et al. 2011). Therefore, it was hypothesized that the priming of the precursor of phenylpropanoid pathway (tyrosine) could activate the PAL. The principal objective of the study was to assess whether exogenous tyrosine could activate PAL to cause over accumulation of secondary metabolites, particularly phenolics involved in $\mathrm{pH}$ tolerance in maize seedlings.

\section{Materials and Methods}

\section{Experiment 1}

Seeds of five maize varieties (E-77, EV-6098, Sadaf, S-2002 and Faisalabad Makki) were obtained from Ayub Agricultural Research Institute (AARI), Faisalabad, Pakistan. Each variety was grown at $25 \pm 2{ }^{\circ} \mathrm{C}$ in a growth chamber. Sand was used as a rooting medium and the $\mathrm{pH}$ of rooting medium was adjusted by provision of Hoagland solution adjusted at $\mathrm{pH}$ 3, 7 and 11. Daily based data for germination percentage was collected for each variety. Eight and twelve days old seedlings were harvested and whole seedlings were used for growth analysis (fresh weight, dry weight and seedling vigour). Two varieties that showed comparable growth in neutral conditions but maximum difference under $\mathrm{pH}$ extremes (low/high) were Sahiwal-2002 and Sadaf. Sadaf with better growth in acidic and alkaline medium was selected as $\mathrm{pH}$ resistant variety and $\mathrm{S}-2002$ as $\mathrm{pH}$ sensitive variety for further experimentation (Fig. S1A-D*).

\footnotetext{
*Further details about the Electronic Supplementary Material (ESM) can be found at the end of the article.
} 


\section{Experiment 2}

A laboratory experiment was conducted in a completely randomized design with three replications. Seeds were primed in 0 and $1 \%$ tyrosine solutions for $24 \mathrm{~h}$ and sown in pots filled with sand. One set was supplemented with neutral, second set with acidic and third set with alkaline Hoagland solution. Eight days old seedlings were harvested as first harvest. Second harvest was taken after the interval of three days. Samples were parted into roots and shoots for further analysis including growth (fresh and dry weight, length and leaf area) and biochemical (total soluble phenolics, flavonoids, CAT and PAL).

\section{Biochemical analysis}

Fresh sample of $0.26 \mathrm{~g}$ was homogenized in $2.5 \mathrm{ml}$ of $75 \mathrm{mM}(\mathrm{pH} 7.0)$ phosphate buffer. Centrifuged at $12,000 \mathrm{~g}$ for $20 \mathrm{~min}$. Then separated the supernatant in microfuge tube and stored in freezer for metabolite phenolics estimation.

\section{Total soluble phenolics}

These were determined following the method of Marinova and Ribarova (2005) sample extract of $10 \mu \mathrm{l}$ was added with $0.25 \mathrm{ml}$ of Folin-Ciocalteu reagent which was 10 times diluted. After $5 \mathrm{~min} 0.25 \mathrm{ml}$ of $7 \%$ sodium carbonate solution was added and mixture was incubated at room temperature for $1 \mathrm{~h}$. The absorbance was measured at $750 \mathrm{~nm}$ using spectrophotometer. Gallic acid was used as standard.

\section{Total flavonoid assay}

Total flavonoid contents were measured following by Ying and Wan (2012). Above-mentioned extract of $0.4 \mathrm{ml}$ was supplied with $0.16 \mathrm{ml}$ of distilled water. Then added $0.12 \mathrm{ml}$ of $5 \% \mathrm{NaNO}_{2}$. After 5 min $0.12 \mathrm{ml}$ of $10 \% \mathrm{AlCl}_{3}$ was added. After 6 min $0.8 \mathrm{ml}$ of $1 \mathrm{M}$ $\mathrm{NaOH}$ was added and made volume up to $2 \mathrm{ml}$ by adding $0.40 \mathrm{ml}$ distilled water. Finally absorbance was measured at $510 \mathrm{~nm}$.

\section{Phenylalanine ammonia-lyase}

Fresh material of $0.2 \mathrm{~g}$ was grinded in $2.5 \mathrm{ml} 150 \mathrm{mM}$ tris-HCl buffer ( $\mathrm{pH}$ 8.0) following Hodgins (1971). Samples were then centrifuged at 12,000 $\mathrm{g}$ for $15 \mathrm{~min}$. Then supernatant was stored in microfuge tube at freezing temperature. The reaction mixture consisted of $1 \mathrm{ml}$ of $3 \mathrm{nM}$ L-Tyrosine solution and $0.5 \mathrm{ml}$ distilled water in $0.1 \mathrm{ml}$ of enzyme extract was followed by immediate readings at $286 \mathrm{~nm}$ for $2 \mathrm{~min}$. Units $/ \mathrm{ml}$ enzyme $=(\triangle \mathrm{A} 286 /$ min test $-\triangle \mathrm{A} 286 / \mathrm{min}$ Blank) (3 dillution factor) (18.5) (0.05). Mixture of $0.1 \mathrm{ml} 150 \mathrm{mM}$ tris- $\mathrm{HCl}$ buffer, $1 \mathrm{ml} 3 \mathrm{mM}$ L-tyrosine and $0.5 \mathrm{ml}$ distilled water was used as a blank. 


\section{Catalase assay}

Fresh $0.5 \mathrm{~g}$ material was grinded in $2.5 \mathrm{ml}$ of $50 \mathrm{mM}$ potassium phosphate buffer. Samples were then centrifuged at $12,000 \mathrm{~g}$ for $15 \mathrm{~min}$. The supernatant was used to measure catalase (CAT) activity by the method proposed by Beers and Sizer (1952). One $\mathrm{ml}$ of $0.05 \mathrm{M}$ potassium phosphate ( $\mathrm{pH} 7.0), 0.5 \mathrm{ml}$ of $0.059 \mathrm{M}$ hydrogen peroxide was used as reaction mixture. Then $0.1 \mathrm{ml}$ of enzyme extract was added and absorbance at $240 \mathrm{~nm}$ for 1 minute was noted. Units $/ \mathrm{mg}=\Delta \mathrm{A} 240 / \mathrm{min} \times 100043.6 \times \mathrm{mg}$ enzyme $/ \mathrm{ml}$ reaction mixture of $1 \mathrm{ml}$ of $0.05 \mathrm{M}$ potassium phosphate $(\mathrm{pH} 7.0)$ and $0.5 \mathrm{ml} 0.059 \mathrm{M}$ hydrogen peroxide solution was used as a blank.

\section{Statistical analysis}

MS-Excel was used for graphical presentation of the data. The analysis of variance (ANOVA) and correlation index was determined using a computer software CoStat cohort 6.4 .

\section{Results}

Statistical analysis showed that variety and priming factors were significant for fresh weight of root and shoot. Furthermore, statistical correlation of fresh weight with different biochemical was significant. It included phenolics (shoot), CAT activity, root and shoot flavonoids (Table S1). Sadaf manifested stability in response to $\mathrm{pH}$ variations in terms of fresh weight whereas; in S-2002, there was a notable decline under acidic and alkaline conditions. Tyrosine priming improved fresh weight of S-2002 raising it up to the level of Sadaf (Fig. S2A-D).

The $\mathrm{pH}$ regimes significantly affected dry weight of seedlings. Correlation of dry weight with root and shoot phenolics and CAT was significant along with root PAL (Table S1). In terms of dry weight both varieties were almost similar in neutral conditions. In acidic and alkaline conditions S-2002 experienced sharp decline of this attribute, whereas, Sadaf variety sustained its dry biomass in both $\mathrm{pH}$ extremes. Tyrosine priming improved dry weight of S-2002 at all pH levels. However Sadaf experienced negligible effect of priming (Fig. S2E-F).

Seed priming with tyrosine significantly improved shoot length in maize under different $\mathrm{pH}$ extremes. Moreover, variety into $\mathrm{pH}$, priming into $\mathrm{pH}$ interactions were also significant along with combined interaction of three factors. Furthermore, correlation matrix for shoot length exhibited positive correlations with PAL, phenolics, and flavonoids of both embryonic parts. Moreover, it positively correlated with root CAT activity (Table S1). Initially both varieties experienced decline of shoots length. Later, in Sadaf there was increase of length in stressed conditions. Similar was the trend of S-2002 in acidic conditions but it failed to show such trend of increase in alkaline rooting medium. Priming improved shoot lengths of both varieties in each harvest with the exception of shoots of S-2002 at second harvest (Fig. S3A-B) stressed with low pH. 
Root lengths manifested positive correlation positive for root and shoot PAL, shoot CAT and shoot phenolics (Table S1). Varietal difference with respect to root length was time based. Formerly, both varieties were with similar lengths of roots that equally decreased under stresses in both varieties. With the course of time, there aroused marked difference amongst varieties where Sadaf proved itself better than S-2002 in low and high $\mathrm{pH}$. Though in control conditions, still there was non-significant difference of root lengths. Priming improved root lengths. This effect was more noticeable in S-2002 than Sadaf (Fig. S3C-D).

For leaf area there was positive correlation with root flavonoids and PAL activity. Moreover, root and shoot phenolics and shoot CAT directly correlated with this attribute (Table S1). Leaf area of both varieties was invariable in response to $\mathrm{pH}$ variations. Both varieties differed for leaf area where Sadaf with greater leaf area was superior than S-2002. Response towards tyrosine priming was positive in both varieties with respect to this attribute (Fig. S3E-F).

Shoot phenolics were directly correlated with PAL activity, root phenolics, fresh and dry weights, root and shoot length and leaf area (Table S1). Sadaf displayed significant reduction in phenolics under unfavourable $\mathrm{pH}$ conditions initially but at final harvest it successfully sustained in all $\mathrm{pH}$ levels. S-2002 exhibited better accumulation of phenolics. Whereas, there was sharp decline in this attribute at medium and high $\mathrm{pH}$ range. Priming improved phenol accumulation maximally in neutral and high pH for S-2002. Whereas, for Sadaf it was maximum in acidic conditions (Fig. S4A-D).

The shoot flavonoids were directly correlated with root flavonoids, fresh weight and shot length (Table S1). Sadaf showed the maximum accumulation at acidic range. With the increase of $\mathrm{pH}$, there was a consistent fall of phenolics contents in shoots though in roots it was comparable in neutral and alkaline conditions. S-2002 was almost unaffected by $\mathrm{pH}$ variation for this attribute. Priming improved phenolics in alkaline conditions of both varieties. Sadaf exhibited marked trend of decline in flavonoid accumulation with increase of $\mathrm{pH}$ in shoots. However, in roots the only significant range was alkaline that was superior for flavonoids. S-2002 failed to show any difference of accumulation with respect to $\mathrm{pH}$ variations. Priming improved flavonoid production only in alkaline conditions in both varieties (Fig. S4E-H).

Shoot length positively correlated with shoot PAL, fresh and dry biomass, leaf area and root length (Table S1). In roots, data of CAT activity manifested significant variance for priming only. Moreover, interactions of $\mathrm{pH}$ with variety and with priming were significant. Furthermore, combined interaction was also significant. Overall both varieties maintained activity of this enzyme at all $\mathrm{pH}$ in both parts at both harvests (Fig. 5A-D).

In shoots of seedlings PAL activity showed direct correlation with root PAL and CAT activity, shoot phenolics, root flavonoids, root and shoot lengths (Table S1). Shoots of both varieties displayed different trends in response to $\mathrm{pH}$ variations. Initially, both varieties experienced decline of PAL activity at high and low $\mathrm{pH}$ at final harvest S-2002 maintained similar trend. Whereas Sadaf grown in alkalinity improved the level of PAL activity even greater than neutral. Priming improved activity of PAL in acidic range and neu- 
tral range in Sadaf. In S-2002 it improved its activity initially in each level but finally in alkaline range.

Root PAL activity directly correlated with shoot CAT activity, phenolics dry weight, lengths and leaf area (Table S1). With the course of time, Sadaf showed a trend of maintenance of PAL activity at variations of $\mathrm{pH}$ such as neutral and high $\mathrm{pH}$. In acidic range, it was with its maximum activity of PAL. S-2002 manifested decline at unfavourable $\mathrm{pH}$. However, it was better than Sadaf in neutral conditions. Priming with tyrosine positively affected maize seedlings for this attribute (Fig. 5E-H).

\section{Discussion}

Influence of environmental variations has been formerly publicized upon plant. In this aspect, growth reduction under high temperature (Mahmood et al. 2010), drought (Azhar et al. 2011) and saline medium (Kaya et al. 2013) has been well studied. Contradictory reports about plant growth at unfavourably low (Fan and Wang 2000) and high pH (Yang et al. 2007; 2008a, c, 2009) are available showing differential pattern varying from species to species. Comprehensive study ranging from acidic to alkaline $\mathrm{pH}$ is scant up to our knowledge. Particularly, the effect of exogenous application of phenylpropanoid pathway precursor as external source of variation, upon PAL activation and related metabolic pool is unrevealed.

In the present work, two varieties that were showing similar biomass in neutral conditions differed in alkaline and acidic range of $\mathrm{pH}$ where Sadaf that got superiority with better growth as compared with S-2002 (Fig. S1). That indicates intra-species differential potential of maize to grow under variant $\mathrm{pH}$ ranges. Moreover, both varieties also differed in their response towards priming, the variety showing lower biomass in $\mathrm{pH}$ extremes surpassed the better variety in term of biomass after tyrosine priming.

Shoot length of both varieties in acidic conditions was greater than neutral one. These findings are in agreement with previous findings (Fan and Wang 2000) where increased plant length was observed at low $\mathrm{pH}$ treatments. Increased shoot length may be related to better accumulation of total soluble phenolics in acidic conditions. For shoot length, $\mathrm{pH}$ could have a more obvious effect than measured one. Though there are some contradictory reports also with reduction of plant height as presented by Balasubramanian et al. (2007). In alkaline conditions generally reduction of shoot length is observed as a sign of commotion faced by plant (Yang et al. 2008b). In the present findings, similar reduction of shoot length was observed only in S-2002 (Fig. S2A-B) indicating its sensitivity towards alkalinity. In contrary, Chude et al. (2004) observed an increased shoot length under similar conditions as was observed in case of Sadaf variety.

Root length is an important feature of plant growth that helps to absorb water from the deeper level of soil (Franco et al. 2006). Reduction in root length lowers accessibility of water that will eventually reduce plant yield. Generally decline in root length is experienced by plants grown under acidic environmental conditions (Kidd and Proctor 2001). That is further correlated with toxicity of $\mathrm{H}^{+}$, consequential membrane damage and cytoplasmic acidification (Babourina et al. 2001). Similar were the findings of present study 
where acidic conditions markedly decreased root lengths in both varieties (Fig. S2C-D). This reduction of root length was more pronounced in S-2002. It showed crucial contribution of $\mathrm{pH}$ for its normal growth. With the course of time effect of acidic rooting medium becomes more critical previously this trend has been observed which consequent into ultimate plant death (Ahmed 2000). Alkalinity generally reduces root length (Yang et al. 2008b; Guan et al. 2009), trend of both varieties was in agreement with former findings. Where alkalinity based lowering of root lengths was greater in S-2002.

Unfavourable environments also lessen leaf area (Yang et al. 2008b; Guan et al. 2009). Sadaf with greater leaf area demonstrated itself better than S-2002 (Fig. S2E-F). Indicating better exposure of Sadaf leaves to solar radiation due to broadening of leaf area, with better photosynthetic activity owing for better yield showing corroboration with previous outcomes (Udensi and Ikpeme 2010).

Overall, tyrosine priming improved maize growth at high/low $\mathrm{pH}$ extremes where sensitive variety responded better than resistant (in terms of root length, shoot length and biomass).

\section{Phenolic metabolism}

Stressed plants need scavenging tactics to lessen hurts endorsed by ROS. These approaches include both enzymatic and non-enzymatic elements. Parallelism in stress tolerance and phenolic metabolism has been reliably stated. These phenolics are synthesized by phenylpropanoid pathway then contribute in plant stress tolerance against heat (Mahmood et al. 2014) and drought (Azhar et al. 2011). Over accumulation of these phenolics contribute structural support improving growth and vigour of plants with better tolerance against stresses (Vogt 2010; Pawlak-Sprada et al. 2011).

Effect of $\mathrm{pH}$ variation upon secondary metabolism range from positive to negative that is in turn species dependent. Generally to cope with adversaries of high (Yadav 2010) and low (Diaz et al. 2001) pH, plants over produce secondary metabolites. Lower accumulation of phenolics is generally correlated by onset of oxidative damage (Mahmood et al. 2014). In present study level of phenolics in both varieties at acidic range showed the potential of both varieties to cope acidity (Fig. S4A-D). Moreover, in alkaline range better accumulation of phenolics in resistant variety (Sadaf) indicated the contribution of phenolics in its comparatively better survival at high $\mathrm{pH}$ than S-2002.

Flavonoids are reported as antioxidant agents by scavenging ROSs, which are functioned by virtue of the number and arrangement of their hydroxyl groups attaches to ring structures. Their ability to act as antioxidants depends on the reduction potentials of their radicals and accessibility of the radicals (Rice-Evans 2001). Better accumulation of phenolics and flavonoid in primed seedlings along with their correlation with growth characteristics may be related with better protection against oxidative damage, screening of harmful radiations, stabilization of sub-cellular structures and improvement in cell water balance as previously reported. Flavonoids are one of the products of phenylpropanoid that sustain osmotic activity of the cell (Wahid et al. 2008) mediating stress tolerance (Mahmood et al. 2014). In the present research, lower accumulation of this metabolite in 
sensitive variety under low and high $\mathrm{pH}$ stress (Fig. S4E-H) showed some relevance with former outcomes. Overall no severe effect of $\mathrm{pH}$ extremes (low and high) was observed upon flavonoid accumulation in the present project. However, the adverse effect of these $\mathrm{pH}$ ranges may be noticeable at some later stages of growth.

\section{Enzymatic activity}

Second component of plant antioxidant system are the enzymes, e.g. SOD (superoxide dismutase), APX (ascorbate peroxidase), GPX (glutathione peroxidase), CAT (catalase), (Zobayed et al. 2005). Parallelism between these enzymes, stress induced lipid peroxidation and stress tolerance has been previously reported (Zobayed et al. 2005; Wu et al. 2012).

The CAT is one of these enzymatic antioxidants (Almeselmani et al. 2006). Activity of CAT showed direct correlation with growth but there was no correlation with products of phenylpropanoid pathway (Table S1) that indicates its possible role in stress tolerance by affecting plant growth. But it did not show direct relevance with the phenolic metabolism.

The PAL catalyzes the key step of the phenylpropanoid pathway (Pawlak-Sprada et al. 2011) justified by positive correlation of PAL with phenolics and flavonoids manifested in present research. That in turn improved growth of both varieties indicated by improved root and shoot length, fresh and dry biomass and leaf area. The expression of PAL like other metabolic enzymes could be regulated allosterically by the substrates and/or the products of the pathway (Huang et al. 2011). Priming with tyrosine (substrate) positively improved PAL activity (Fig. 1E-H). Thereafter activation of PAL enzyme resulted into the overproduction of phenolics and flavonoids that augment stress tolerance against $\mathrm{pH}$ stressors. That accretion of the products of phenylpropanoid pathway added structural sustenance, better growth and stress tolerance confirming previous findings (Vogt 2010; Pawlak-Sprada et al. 2011).

Tyrosine priming improved phenolic metabolism in terms of PAL activity and associated over-accumulation of total soluble phenolics and flavonoids along with improved growth. Tolerance potential of maize varieties correlated with phenolics metabolism. Moreover, the tolerance ability of the maize to $\mathrm{pH}$ stress was partly related to the increased activity of CAT that directly correlated with improved growth though lack direct link with phenolics metabolism. In the nutshell, tolerance potential of maize varieties correlated with phenolic metabolism, i.e. PAL activity and the accumulation of its products in terms of phenolics and flavonoids in the sensitive variety under low and high $\mathrm{pH}$ stress.

An extended experiment leading to the yield of crop is desired to be conducted for further confirmation of the hypothesis of this work. Furthermore, metabolic profiling of maize plants growing at varying $\mathrm{pH}$ levels may provide deeper insight to explore the contribution of phenolic metabolism in these conditions. Moreover, it is needed to find out some cheap source of tyrosine. So far it could be recommended to farmers to get economic solution for increasing geographical range of growing areas of maize or other crops. 


\section{Acknowledgement}

The data of the paper is the part of the M.Sc. thesis of the third author (Ayesha Ashraf).

\section{References}

Ahmed, M.F. 2000. Soil environment and arsenic contamination of groundwater in Bangladesh. Proc. Int. Conf. on Geo-environment. Sultan Qabus University. Muscat, Oman, 1:256-264.

Almeselmani, M., Deshmukh, P.S., Sairam, R.K., Kushwaha, S.R., Singh, T.P. 2006. Protective role of antioxidant enzymes under high temperature stress. Plant Sci. 71:382-388.

Azhar, N., Hussain, B., Ashraf, M.Y., Abbasi, K.Y. 2011. Water stress mediated changes in growth, physiology and secondary metabolites of desi ajwain (Trachyspermum ammi L.). Pakistan J. of Bot. 43:15-19.

Babourina, O., Hawkins, B., Lew, R.R., Newman, I., Shabala, R. 2001. K ${ }^{+}$transport by Arabidopsis root hairs at low pH. Aust. J. Plant Physiol. 28:635-641.

Balasubramanian, G., Udayasoorian, C., Prabu, P.C. 2007. Effects of short-term exposure of simulated acid rain on the growth of Acacia nilotica. J. of Tropical Forest Sci. 19:198.

Beers, R.F., Sizer, I.W. 1952. A spectrophotometric method for measuring the breakdown of hydrogen peroxide by catalase. J. of Biol. Chem. 41:133-140.

Chude, V.O., Malgwi, W.B., Amapu, I.Y., Ano, O.A. 2004. Manual on soil fertility assessment. Published by Federal Fertilizer Department in collaboration with National Special Programme for Food Security. Abuja, Nigeria, pp. 32-38.

de Lacerda, C., Cambraia, J., Oliva, M., Ruiz, H., Prisco, I. 2003. Solute accumulation and distribution during shoot and leaf development in two sorghum genotypes under salt stress. Environ. and Exp. Bot. 49:107120 .

Deng, W., Qiu, S.W., Liang, Z.W. 2006. Background of Regional Eco-environment in Daan Sodic Land Experiment Station of China. Science and Technology Press. Beijing, China.

Diaz, J., Bernel, A., Porner, F., Merino, F.M. 2001. Induction of shikmate dehydrogenase and peroxidase in pepper (Capsicum annum L.). Seedling in response to copper stress and in relation to lignification. Plant Sci. 161:179-188.

Fan, H.B., Wang, Y.H. 2000. Effects of simulated acid rain on germination, foliar damage, chlorophyll contents and seedling growth of hardwood species growing in China. Forest Ecology Management 126:321-329.

Foy, R.H. 1992. A phosphorus loading model for Northern Irish Lakes. Water Resources 26:633-638.

Foyer, C.H., Noctor, G. 1992. Oxidant and antioxidant signalling in plants: a re-evaluation of the concept of oxidative stress in a physiological. Plant Cell Environ. 28:1056-1071.

Franco, J.A., Martínez-Sánchez, J.J., Fernández, J.A., Bañón, S.A. 2006. Selection and nursery production of ornamental plants for landscaping and xerogardening in semi-arid environments. J. of Horticulture Sci. and Biotechnol. 81:3-17.

Goodey, N.M., Benkovic, S.J. 2008. Allosteric regulation and catalysis emerge via a common route. Natural Chem. and Biol. 4:474-482.

Guan, B., Zhou, D., Zhang, H., Tian, Y., Japhet, Y. 2009. Germination responses of Medicago ruthenica seeds to salinity, alkalinity, and temperature. J. of Arid Environments 73:135-138.

Hodgins, D.S. 1971. Yeast phenylalanine ammonia-lyase purification, properties, and the identification of catalytically essential dehydroalanine. J. of Biol. Chem. 246:2977-2985.

Huang, J., Song, Z., Liu, Y., Jin, Q., Wu, X., Cai, C., Wang, X. 2011. Enzymatic synthesis of monoacylglycerol citrate optimized by response surface methodology. Eur. J. of Lipid Sci. and Technol. 113:609-615.

Kaya, C., Aydemir, S., Sonmez, O. 2013. Regulation of growth and some key physiological processes in saltstressed maize (Zea mays L.) plants by exogenous application of asparagine and glycerol. Acta Botanica 72:157-168.

Kidd, P.S., Proctor, J. 2001. Why plants grow poorly on very acid soils: are ecologists missing the obvious? J. of Exp. Bot. 52:791-799. 
Mahmood, S., Wahid, A., Javed, I.F., Basra, S.M.A. 2010. Heat stress effects on forage quality characteristics of maize (Zea mays) cultivars. Int. J. of Agric. and Biol. 12:701-706.

Mahmood, S., Parveen, A., Hussain, I., Javed, S., Iqbal, M. 2014. Possible involvement of secondary metabolites in the thermotolerance of maize seedlings. Int. J. of Agric. and Biol. 16:1075-1082.

Marinova, D.F., Ribarova, A.M. 2005. Total phenolics and total flavonoids in Bulgarian fruits and vegetables. J. of Universal Chemical Technol. and Metallurgy 40:255-260.

Pawlak-Sprada, S., Arasimowicz-Jelonek, M., Podgórska, M., Deckert, J. 2011. Activation of phenylpropanoid pathway in legume plants exposed to heavy metals. Part I. Effects of cadmium and lead on phenylalanine ammonia-lyase gene expression, enzyme activity and lignin content. Acta Biochimica Polonica 2:211-216.

Raes, J., Rohde, A., Christensen, J.H., Van de Peer, Y., Boerjan, Y. 2003. Genome-wide characterization of the lignification toolbox in Arabidopsis. Plant Physiol. 133:1051-1071.

Rice-Evans, C. 2001. Flavonoid antioxidants. Current Medical Chem. 8:797-807.

Samac, D.A., Tesfaye, M. 2003. Plant improvement for tolerance to aluminum in acid soils: a review. Plant Cell Tissue and Org. Cult. 75:189-207.

Tzin, V., Galili, G. 2010. The biosynthetic pathways for shikmate and aromatic amino acids in Arabidopsis thaliana. The Arabidopsis Book: e0132. doi: 10.1199/tab.0132. [PMC free article] [PubMed]

Udensi, O., Ikpeme, E.V. 2010. Correlation and path coefficient analyses of seed yield and its contributing traits in Cajanus cajan (L.) Mill sp. Amm. J. of Exp. Agric. 2:351-358.

Vogt, T. 2010. Phenylpropanoid biosynthesis. Mol. Plant 3:2-20.

Wahid, A., Farooq, M., Basra, S.M.A., Rasul, E. 2008. Metabolites accumulation in plants under high temperature stress. In: Proc. Int. Workshop on Carbon and Water Exchange in Plants under Changing Climatic Conditions. Nov. 5-6, 2007. Pir Mehr Ali Shah Arid Agriculture University. Rawalpindi, Pakistan. pp. $103-115$.

Wu, G.Q., Zhang, L.N., Wang, Y.Y. 2012. Response of growth and antioxidant enzymes to osmotic stress in two different wheat (Triticum aestivum L.) cultivar seedlings. Plant Soil Environ. 58:534-539.

Yadav, S.K. 2010. Heavy metals toxicity in plants: an overview on the role of glutathione and phytochelatins in heavy metal stress tolerance of plants. South Afr. J. Bot. 76:167-179.

Yang, C., Chong, J., Kim, C., Li, C., Shi, D., Wang, D. 2007. Osmotic adjustment and ion balance traits of an alkali resistant halophyte Kochia sieversiana during adaptation to salt and alkali conditions. Plant and Soil 294:263-276.

Yang, C., Jianaer, A., Li, C., Shi, C., Wang, D. 2008a. Comparison of the effects of salt-stress and alkali-stress on the photosynthetic production and energy storage of an alkali-resistant halophyte Chloris virgata. Photosynthetica 46:273-278.

Yang, C.W., Shi, D.C., Wang, D.L. 2008b. Comparative effects of salt and alkali stresses on growth, osmotic adjustment and ionic balance of an alkali-resistant halophyte Suaeda glauca (Bge.). Plant Growth Regulation 56:179-190.

Yang, C., Wang, P., Li, C., Shi, D., Wang, D. 2008c. Comparison of effects of salt and alkali stresses on the growth and photosynthesis of wheat. Photosynthetica 46:107-114.

Yang, C., Xu, H., Wang, L., Liu, J., Shi, D., Wang, D. 2009. Comparative effects of salt stress and alkali-stress on the growth, photosynthesis, solute accumulation, and ion balance of barley plants. Photosynthetica 47:79-86.

Ying, C., Wan, D. 2012.Quantitative determination of total and individual flavonoids in stems and leaves of Buddleja davidii and Buddleja albiflora. Pharmacognosy 8:273-279.

Zobayed, S.M.A., Afreen, F., Kozai, T. 2005. Temperature stress can alter the photosynthetic efficiency and secondary metabolite concentrations in St. John's wort. Plant Physiol. and Biochem. 43:977-984. 


\section{Electronic Supplementary Material (ESM)}

Electronic Supplementary Material (ESM) associated with this article can be found at the website of CRC at http://www.akademiai.com/content/120427/

Electronic Supplementary Table S1. Correlation matrix of two maize (Zea mays L.) varieties growing in different $\mathrm{pH}(3.7$ and 11) regimes with differential potential of stress tolerance

Electronic Supplementary Figure S1. Comparative response of five maize (Zea mays L.) varieties growing in different $\mathrm{pH}(3,7$ and 11) regimes. Analyzed individually in triplicate $(\mathrm{n}=1 \times 3 \times 3)$. Error bars indicate \pm STDEV and LSD (at $p \leq 0.05$ ) least significant difference

Electronic Supplementary Figure S2. Effect of tyrosine priming upon the root and shoot dry weight and root fresh weight of two maize (Zea mays L.) varieties (differing in stress tolerance), grown in three $\mathrm{pH}$ (3.7 and 11) regimes at two harvests (left and right). Error bars indicate \pm STDEV and LSD (at $p \leq 0.05$ ) least significant difference for priming factor

Electronic Supplementary Figure S3. Effect of tyrosine priming upon the leaf area and root and shoot length of two maize (Zea mays L.) varieties (differing in stress tolerance), grown in three $\mathrm{pH}$ (3.7 and 11) regimes at two harvests (left and right). Error bars indicate \pm STDEV and LSD (at $p \leq 0.05$ ) least significant difference for priming factor

Electronic Supplementary Figure S4. Effect of tyrosine priming upon total soluble phenolics (TSP) and flavonoids (FLV) of two maize (Zea mays L.) varieties (differing in stress tolerance), grown in three $\mathrm{pH}$ (3.7 and 11) regimes at two harvests (left and right). Error bars indicate \pm STDEV and LSD (at $p \leq 0.05$ ) least significant difference for priming factor

Electronic Supplementary Figure S5. Effect of tyrosine priming upon enzymatic activities of two maize (Zea mays L.) varieties (differing in stress tolerance), grown in three $\mathrm{pH}$ (3.7 and 11) regimes at two harvests (left and right). Error bars indicate \pm STDEV and LSD (at $p \leq 0.05$ ) least significant difference for priming factor 